\title{
Modern Aspects of Superconductivity
}

Theory of Superconductivity 


\section{Modern Aspects of Superconductivity Theory of Superconductivity}

\section{Sergei Kruchinin}

Bogolyubov Institute for Theoretical Physics, Kiev, Ukraine

Hidemi Nagao \& Shigeyuki Aono

Kanazawa University, Japan 


\section{Published by}

World Scientific Publishing Co. Pte. Ltd.

5 Toh Tuck Link, Singapore 596224

USA office: 27 Warren Street, Suite 401-402, Hackensack, NJ 07601

UK office: 57 Shelton Street, Covent Garden, London WC2H 9HE

\section{British Library Cataloguing-in-Publication Data}

A catalogue record for this book is available from the British Library.

\section{MODERN ASPECTS OF SUPERCONDUCTIVITY \\ Theory of Superconductivity}

Copyright (C) 2011 by World Scientific Publishing Co. Pte. Ltd.

All rights reserved. This book, or parts thereof, may not be reproduced in any form or by any means, electronic or mechanical, including photocopying, recording or any information storage and retrieval system now known or to be invented, without written permission from the Publisher.

For photocopying of material in this volume, please pay a copying fee through the Copyright Clearance Center, Inc., 222 Rosewood Drive, Danvers, MA 01923, USA. In this case permission to photocopy is not required from the publisher.

ISBN-13 978-981-4261-60-9

ISBN-10 981-4261-60-2

Typeset by Stallion Press

Email: enquiries@stallionpress.com

Printed in Singapore. 


\section{The page is intentionally left blank}


"The single reason for our inability to treat the problems of superconductivity consists in the absence of a sufficient imagination."

— Richard P. Feynman 


\section{The page is intentionally left blank}




\section{Preface}

Studies of superconductivity theory are among the most fruitful and promising trends in the theoretical physics of condensed matter, since superconductivity remains one of the most interesting research areas in physics.

The goal of this book is to give a representation of certain modern aspects of superconductivity. We discuss important aspects of the theory of superconductivity, such as the nature of high- $T_{c}$ superconductivity, two-gap superconductivity, room-temperature superconductivity, mesoscopic superconductivity, the pairing state and the mechanism of cuprate high- $T_{c}$ superconductivity.

In Chap. 1, we consider the field-theoretical method of superconductivity and discuss the basic idea of superconductivity and the elaboration of the Ginzburg-Landau and Bardeen-Cooper-Schrieffer theories in the frame of many-particle quantum field theory.

In Chap. 2, we consider the structures of high- $T_{c}$ superconductors, phase diagrams and the problem of pseudogaps, and analyze the mechanisms of superconductivity. We present general arguments regarding the pairing symmetry in cuprate superconductors and investigate their thermodynamical properties within the spin-fluctuation mechanism of superconductivity, by using the method of functional integrals.

Chapter 3 concentrates on two-band and multiband superconductivity. We consider the physical properties of the superconductor $\mathrm{MgB}_{2}$ and use our two-band model to explain the two coupled superconductor gaps of $\mathrm{MgB}_{2}$. To study the effect of the increasing $T_{c}$ in $\mathrm{MgB}_{2}$, we use the renormalization-group approach and phase diagrams. In the field of superconductivity we meet the problem-maximum, which consists in the creation of room-temperature superconductors. We consider this problem in our book and make some recommendations on the search for these superconductors. 
Chapter 4 deals with the problem of mesoscopic superconductivity. We consider the two-band superconductivity in ultrasmall grains, by extending the Richardson exact solution to two-band systems, and develop the theory of interactions between nanoscale ferromagnetic particles and superconductors. The properties of nanosize two-gap superconductors and the Kondo effect in superconducting ultrasmall grains are investigated as well.

We also consider the ideas of quantum computing and quantum information in mesoscopic circuits. The theory of the Josephson effect is presented, and its applications to quantum computing are analyzed.

This book deals with a wide scope of theoretical and experimental topics in superconductivity, and has been written for advanced students and researchers in that field.

S. Kruchinin, H. Nagao and S. Aono Kiev, Kanazawa, July 2009 


\section{Contents}

Preface vii

1. Theory of Superconductivity 1

1.1 Introduction . . . . . . . . . . . . . . . . 1

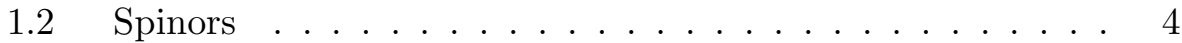

1.2.1 Spinor ............... . . 6

1.2.2 Noether theorem and Nambu-Goldstone

theorem . . . . . . . . . . . . . 7

1.3 Propagator .................. 10

1.3.1 Hamiltonian .............. . 11

1.4 Noninteracting . . . . . . . . . . . . . . . 12

1.5 Interacting . . . . . . . . . . . . . . . . . 15

1.5.1 Unrestricted Hartree-Fock (HF) . . . . . . . . . . . 19

1.5.2 Gap equation for superconductivity . . . . . . . . 20

1.6 Illustrative Example, Critical Temperature . . . . . . . . . 21

1.6.1 Bond alternation . . . . . . . . . . . . 21

1.6.2 Deformation energy ............ . . 23

1.6.3 Polyacene, gap equation, critical temperature . . . . 24

1.6.4 Conclusion . . . . . . . . . . . . . . 27

1.7 Linear Response Magnetic Resonance

in Normal and Superconducting Species;

Spin-lattice Relaxation Time . . . . . . . . . . . . 28

1.7.1 Introduction . . . . . . . . . . . . . . 28

$1.7 .2 T_{1}$ in NMR . . . . . . . . . . . . 31

1.7.3 Theory with Green's function . . . . . . . . . 32

1.7.4 Noninteracting . . . . . . . . . . . . 35

1.7.5 Interacting; normal ............ 36 
1.8 Interacting; Superconductor . . . . . . . . . . . . . . . . . . 39

1.8.1 The extended Nambu spinor . . . . . . . . . . 39

1.8.2 Green's function ... . . . . . . . . . . . 40

1.8.3 Spin dynamics . . . . . . . . . . . . . . . . . . . . . . . . . . 41

1.8.4 Conclusion . . . . . . . . . . . . . . 42

1.9 Ginzburg-Landau Theory from the BCS Hamiltonian . . . 43

1.9.1 BCS theory .............. . . 44

1.9.2 Hubbard-Stratonovitch transformation . . . . . . 46

1.9.3 Fourier transform ... . . . . . . . . . . 48

1.9.4 Nambu spinor . . . . . . . . . . . . . . 48

1.9.5 Critical temperature............. 51

1.9.6 Temperature dependence of $\phi$. . . . . . . . . 52

1.9.7 Dynamics of the boson field; symmetry breaking . . 53

1.9 .8 Instability . . . . . . . . . . . . . . 55

1.9 .9 Supersymmetry . . . . . . . . . . . . 56

1.9.10 Towards the Ginzburg-Landau equation . . . . . . 58

1.9.11 Discussion .............. . 59

2. Physics of High- $T_{c}$ Superconductors $\quad 61$

2.1 Introduction . . . . . . . . . . . . . . . 61

2.2 History of the Development of Superconductivity . . . . . . 64

2.3 Structural Properties of High-Temperature

Superconductors . . . . . . . . . . . . . . . 65

2.3.1 Phase diagram of cuprate superconductors . . . . . 69

2.4 Mechanisms of Pairing of HTSCs . . . . . . . . . . . . . 72

2.4.1 Specific mechanisms of pairing in superconductivity 72

2.4.2 Magnetic mechanism of pairing . . . . . . . . 74

2.4.3 Exciton mechanism of pairing ........ . 75

2.4.4 Anharmonic model and superconductivity . . . . . 76

2.4.5 Van Hove singularities . . . . . . . . . . 77

2.4.6 Plasmon mechanism of pairing . . . . . . . . . . 78

2.4.7 Bipolaronic mechanism of superconductivity . . . 78

2.5 Symmetry of Pairing in Cuprate Superconductors . . . . . . 80

2.5.1 The superconductor's order parameter . . . . . . 80

2.5.2 Classification of the superconductor's order parameter by the representations of symmetry groups . . 82

2.6 Experimental Studies of the Symmetry

of the Superconducting Order Parameter . . . . . . . . . . . 84

2.6.1 Measurements of the Josephson tunnel current . . . 84 
2.6.2 Measurements of the quantization of a flow

by the technique of the three-crystal unit . . . . . 86

2.7 Thermodynamics of the $d$ Pairing

in Cuprate Superconductors . . . . . . . . . . . . . . . 88

2.7.1 Introduction . . . . . . . . . . . . . . . 88

2.7.2 Antiferromagnetic spin fluctuations in HTSCs . . . 89

2.7.3 Continual model of antiferromagnetic spin

fluctuations . . . . . . . . . . . . . . . . 92

2.7.4 Equation for the superconducting gap . . . . . . . 97

2.7.5 Thermodynamic potential of antiferromagnetic

spin fluctuations . . . . . . . . . . . . . . . . 101

2.7.6 Heat capacity of the $d$ pairing . . . . . . . . . . 103

2.7.7 Heat capacity jump in superconductors . . . . . . . 104

2.8 Summary . . . . . . . . . . . . . . . . . 106

3. Multiband Superconductivity 108

3.1 Introduction . . . . . . . . . . . . . . . . . 108

3.2 Multiband Hamiltonian . . . . . . . . . . . . . . . 110

3.2.1 Hamiltonian . . . . . . . . . . . . . . . . . . . 110

3.2.2 Two-particle Green function . . . . . . . . . . . . . 112

3.2.3 Traditional superconductivity . . . . . . . . . . . . 114

3.2.4 Copper oxides . . . . . . . . . . . . . . . . 115

3.2.5 Cooperative mechanism . . . . . . . . . . . . 115

3.2.6 Room-temperature superconductors . . . . . . . . 116

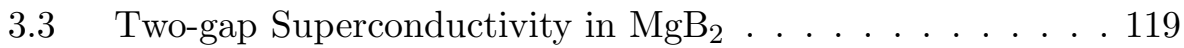

3.3.1 The physical properties of $\mathrm{MgB}_{2}$. . . . . . . . 119

3.3.2 Theoretical model . . . . . . . . . . . . . . . . 122

3.3.3 Superconductivity in $\mathrm{MgB}_{2} \ldots \ldots$. . . . . . . . . . 124

3.4 Theoretical Studies of Multiband Effects

in Superconductivity by Using

the Renormalization Group Approach . . . . . . . . . . 127

3.4.1 Theoretical model . . . . . . . . . . . . . . . 128

3.4.2 Renormalization group approach . . . . . . . . . 130

3.4.3 Vertex correction and response function

for Cooper pairs . . . . . . . . . . . . . 131

3.4.4 Renormalization equation . . . . . . . . . . . 132

3.4.5 Phase diagrams . . . . . . . . . . . . . 133

4. Mesoscopic Superconductivity 135

4.1 Introduction . . . . . . . . . . . . . . . . . 135 
4.2 Nanosize Two-Gap Superconductivity . . . . . . . . . . . . 137

4.2.1 Hamiltonian for nanosize grains . . . . . . . . . 138

4.2.2 Path-integral approach . . . . . . . . . . . . . 139

4.2.3 Condensation energy . . . . . . . . . . . . 141

4.2.4 Critical level spacing . . . . . . . . . . . . . 142

4.2.5 Parity gap . . . . . . . . . . . . . . 143

4.3 Exact Solution of Two-Band Superconductivity

in Ultrasmall Grains . . . . . . . . . . . . . . . . . . . . . 145

4.3.1 Exact solution for two-band superconductivity . . . 146

4.3.2 Hamiltonian . . . . . . . . . . . . . . . . 146

4.3.3 Exact solution . . . . . . . . . . . . . . 148

4.3.4 Preprocessing for numerical calculations . . . . . 151

4.3.5 Results and discussion . . . . . . . . . . . 153

4.3.6 Pair energy level . . . . . . . . . . . . . 153

4.3.7 Condensation energy . . . . . . . . . . . . . 154

4.3.8 Parity gap . . . . . . . . . . . . . . . 155

4.4 Kondo Effect Coupled to Superconductivity . . . . . . . . . 157

4.4.1 Kondo regime coupled to superconductivity . . . . 158

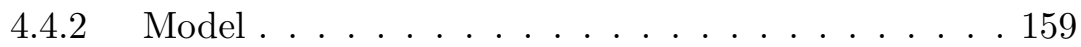

4.4.3 Mean-field approximation . . . . . . . . . . 160

4.4.4 Critical level spacing in the Kondo effect . . . . . . 161

4.4.5 Kondo effect coupled to superconductivity . . . . . 162

4.4.6 Exact solution for the Kondo regime . . . . . . . . 163

4.5 Interaction of Nanoscale Ferromagnetic Granules in London Superconductors . . . . . . . . . . . . . 166

4.5.1 Magnetic field of ferromagnetic inclusions in a London superconductor . . . . . . . . . . . 168

4.5.2 Magnetic field of ferromagnetic quantum dots in a superconducting nanocomposite material . . 169

4.5.3 Interaction energy of quantum dots in a superconducting nanocomposite material . . . 170

4.5.4 Spin-orientation phase transitions in a nanocomposite material with arrays of ferromagnetic quantum dots . . . . . . . . . . . . . . 171

4.6 Spin-Orientation Phase Transitions in a Two-Dimensional Lattice of Ferromagnetic Granules in a London-Type Superconductor . . . . . . . . . 174

4.7 Quantum Computer on Superconducting Qubits . . . . . 181 4.7.1 Principle of quantum computers . . . . . . . . . 181 
4.7.2 Superconducting qubits . . . . . . . . . . . . . 182

4.7 .3 Josephson effect . . . . . . . . . . . . . . . . . 183

Summary and Conclusions

Appendix A: Two-particle Green Function for Multiband Superconductors

Appendix B: A Solution Method for the Ferromagnetic

Granules in London Superconductors

Bibliography 203

Index 